\title{
P01-140
}

\section{COMPARISON OF SLEEP OUTCOMES IN GENERALIZED ANXIETY DISORDER FOLLOWING TREATMENT WITH PREGABALIN OR VENLAFAXINE-XR}

\author{
B. Herman ${ }^{1}$, M. Mychaskiw ${ }^{2}$, F. Mandel ${ }^{1}$ \\ ${ }^{1}$ Medical Division, Pfizer, Inc., ${ }^{2}$ Global Outcomes Research, Pfizer Inc., New York, USA
}

Objective: To evaluate the differential effect of pregabalin and venlafaxine-XR versus placebo on sleep outcomes in non-depressed outpatients with generalized anxiety disorder (GAD).

Methods: This secondary analysis was based on data from a double-blind trial in which adults who met DSM-IV criteria for GAD were randomized to 8-weeks of flexible-dose treatment with pregabalin (300-600 mg/d; $N=121$; baseline HAM-A=27.6), venlafaxineXR (75-225 mg/d: N=125; HAM-A=27.4), or placebo (PBO; N=128; HAM-A=26.8). Sleep was evaluated at baseline, weeks 4, 8, and endpoint using the Medical Outcomes Study (MOS) Sleep Scale, including an overall sleep problems index (SPI), as well as subscale scores such as sleep disturbance.

Results: At baseline, $64 \%$ of patients with GAD met MOS-Sleep scale criteria for insomnia. Treatment with pregabalin was associated with significant endpoint improvement in the MOS-SPI compared to both placebo $(-18.1$ vs. $-10.5 ; P<0.01)$ and venlafaxine-XR $(-10.1: P<0.01)$. Treatment with pregabalin was associated with significant endpoint improvement in the MOS-sleep disturbance score compared to both placebo $(-22.2$ vs. $-12.0 ; P<0.001)$ and venlafaxine-XR $(-11.6: P<0.001)$. While somnolence as a treatment-emergent adverse event occurred more frequently on pregabalin $(9.1 \%)$ compared to venlafaxine-XR (4.8\%) and placebo $(2.3 \%)$, overall, patients treated with pregabalin reported greater reduction in daytime sleepiness compared to venlafaxineXR on the MOS-daytime sleepiness sub-scale (-9.7 vs. -5.8).

Conclusions: Treatment of moderate-to-severe GAD with pregabalin was associated with significantly better sleep outcomes compared to both placebo and venlafaxine-XR. Improvement in anxiety on pregabalin was associated with reduction in daytime sleepiness. 